\title{
Self-pulsing in Fabry-Perot lasers: An analytic scenario
}

\author{
Luigi A. Lugiato and Franco Prati $\odot^{*}$ \\ Dipartimento di Scienza e Alta Tecnologia, Università dell'Insubria, Via Valleggio 11, 22100 Como, Italy
}

(Received 3 June 2019; published 2 December 2019)

\begin{abstract}
We analyze multimode instabilities in the Fabry-Perot laser. It is well known that in the case of a ring cavity the multimode instability can arise only when the pump parameter is several times above threshold. We focus on the parametric conditions that allow for the adiabatic elimination of the atomic polarization only. Under such conditions no multimode instability is possible in the ring configuration. By investigating the stability of the stationary solutions in a fully analytical manner, we demonstrate that, on the contrary, in the Fabry-Perot case a multimode instability can arise very close to threshold and is governed by a remarkably simple formula. Numerical solutions of the dynamical equations confirm this scenario and describe the self-pulsations generated by this instability.
\end{abstract}

DOI: 10.1103/PhysRevResearch.1.032029

The usefulness of the investigations on instabilities and pattern formation in nonlinear optical systems is demonstrated, for example, by the realization of microresonatorbased Kerr frequency combs, especially those generated by cavity solitons [1,2]. Their behavior is governed by an equation [called the Lugiato-Lefever equation (LLE) in the following] formulated in 1987 [3], 20 years before the discovery of Kerr frequency combs; for a discussion of the LLE and its connections with microresonator frequency combs, see Refs. [4-7]. Combined with the ability to miniaturize and integrate on chip, soliton Kerr frequency combs have already found applications, e.g., in dual-comb spectroscopy, frequency synthesis, low-noise microwave generation, laser frequency ranging, and astrophysical spectrometer calibration $[5,7]$.

These works concern systems without population inversion, that display optical bistability. The first multimode optical instabilities that were predicted [8-10] were concerned, instead, with ring lasers. The linear stability analysis of the stationary solution of the Maxwell-Bloch equations under resonant conditions [6] for the ring laser shows that these instabilities arise at least nine times above threshold, a feature that severely limits the practical interest of the self-pulsing generated by them.

According to common wisdom, multimode instabilities in lasers are favored by the presence of an inhomogeneity in the gain medium, that weakens the competition among cavity modes leading to single-mode behavior. One example is inhomogeneous broadening (spectral hole burning) and, as a matter of fact, the instability threshold in inhomogeneously broadened lasers can be substantially lower $[6,11,12]$.

\footnotetext{
*franco.prati@uninsubria.it

Published by the American Physical Society under the terms of the Creative Commons Attribution 4.0 International license. Further distribution of this work must maintain attribution to the author(s) and the published article's title, journal citation, and DOI.
}

Another example is Fabry-Perot lasers, because different cavity modes interact with atoms in different spatial positions owing to the standing wave configuration (spatial hole burning). A good deal of theoretical and experimental work has been done in order to correctly understand multimode emission in Fabry-Perot cavity lasers, starting with the approximate model provided by the Tang-Statz-deMars equations [13]. We refer the interested reader to Refs. [14-17] and references therein. However, to our knowledge, there is no systematic study of instabilities in Fabry-Perot lasers in the framework of the fundamental Maxwell-Bloch equations [6]. The investigations in the literature (see, e.g., Refs. [18,19]) offer only numerical solutions of these equations for selected values of the parameters. A linear stability analysis is lacking, and there is no picture of the domain in parameter space that is most favorable for self-pulsing.

In this Rapid Communication we start from the equations that govern the dynamics of Fabry-Perot lasers [20] and demonstrate that for what concerns multimode instabilities the most relevant scenario arises when the longitudinal relaxation rate $\gamma_{\|}$of population inversion is much smaller than the transverse relaxation rate $\gamma_{\perp}$ of the atomic polarization, in sharp contrast with the ring cavity case. We focus on the resonant configuration and on the parametric conditions that allow for the adiabatic elimination of the atomic polarization (for example, the conditions that characterize class-B lasers) and show analytically that the linear stability analysis leads to a remarkably simple formula for the instability boundary. Our analytical and numerical results demonstrate that the multimode instability and the consequent self-pulsing arise very close to the laser threshold. We do expect that this surprising result will stimulate important experimental work.

The complexity of the Fabry-Perot case is due to the fact that there are two field envelopes, one propagating in the forward and the other in the backward direction. In Ref. [20] we consider a cavity that occupies the interval $0 \leqslant z \leqslant L$ and show that, by considering the symmetrically doubled cavity in the interval $-L \leqslant z \leqslant L$ and appropriately defining the dynamical variables in the additional interval $-L \leqslant z \leqslant 0$, it 
is possible to reformulate the dynamical equations in terms of the forward propagating envelope only [21],

$$
\begin{aligned}
& \tilde{c} \frac{\partial F_{F}(z, t)}{\partial z}+\frac{\partial F_{F}(z, t)}{\partial t} \\
& =-k\left[F_{F}(z, t)-\frac{A}{2 \pi} \int_{-\pi}^{\pi} d \varphi e^{-i \varphi} P(z, \varphi, t)\right], \\
& \gamma_{\perp}^{-1} \frac{\partial P(z, \varphi, t)}{\partial t}=\left[F_{F}(z, t) e^{i \varphi}+F_{F}(-z, t) e^{-i \varphi}\right] \\
& \quad \times D(z, \varphi, t)-P(z, \varphi, t), \\
& \gamma_{\|}^{-1} \frac{\partial D(z, \varphi, t)}{\partial t}=-\frac{1}{2}\left\{\left[F_{F}(z, t) e^{i \varphi}+F_{F}(-z, t) e^{-i \varphi}\right]\right. \\
& \left.\quad \times P^{*}(z, \varphi, t)+\text { c.c. }\right\}-[D(z, \varphi, t)-1],
\end{aligned}
$$

where $F_{F}(z, t)$ denotes the forward propagating envelope, $P(z, \varphi, t)$ and $D(z, \varphi, t)$ indicate the normalized atomic polarization and population inversion of the two-level atoms, respectively, and $\tilde{c}=c / n_{B}$ is the light velocity in the medium, with $n_{B}$ being the background refractive index. We assume that the medium fills the cavity; the case where the medium occupies only part of the cavity will be considered in Ref. [22]. The cavity damping rate $k$ is defined as $k=\tilde{c} T / 2 L$, where $T \ll 1$ is the intensity transmissivity coefficient of the cavity mirrors, and $A$ is the pump parameter with $A=2 g L / T$, where $g$ is the atom-field coupling constant [6]. Time is indicated as $t$, while there are two distinct spatial variables, the slow spatial variable $z$ (which varies on the scale of the cavity length) and the fast spatial variable $\varphi=\omega_{0} z / \tilde{c}$ which varies from $-\pi$ to $+\pi$ and is related to the wavelength scale $[23,24]$; $\omega_{0}$ is the cavity frequency which coincides with the atomic transition frequency. In the additional interval $-L \leqslant z \leqslant 0$ the quantities $F_{F}(z, t), P(z, \varphi, t)$, and $D(z, \varphi, t)$ are defined as

$$
\begin{aligned}
F_{F}(z, t) & =F_{B}(-z, t), \\
P(z, \varphi, t) & =P(-z,-\varphi, t), \\
D(z, \varphi, t) & =D(-z,-\varphi, t),
\end{aligned}
$$

where $F_{B}(z, t)$ is the backward propagating envelope. As a consequence of Eq. (2a), the boundary condition for $F_{F}(z, t)$ in the interval $-L \leqslant z \leqslant L$ is [20]

$$
F_{F}(L, t)=F_{F}(-L, t) .
$$

If we expand $F_{F}(z, t), P(z, \varphi, t)$, and $D(z, \varphi, t)$ as follows,

$$
\begin{gathered}
F_{F}(z, t)=\sum_{n=-\infty}^{+\infty} \tilde{f}_{n}(t) e^{i \frac{\pi n}{L} z-i \alpha_{n} t}, \\
P(z, \varphi, t)=\sum_{n=-\infty}^{+\infty} \tilde{p}_{n}(\varphi, t) e^{i \frac{\pi n}{L} z-i \alpha_{n} t}, \\
D(z, \varphi, t)=\sum_{n=-\infty}^{+\infty} d_{n}(\varphi, t) e^{i \frac{\pi n}{L} z},
\end{gathered}
$$

where

$$
\alpha_{n}=\frac{\pi \tilde{c}}{L} n,
$$

and insert Eqs. (4a)-(4c) into Eqs. (1a)-(1c), we obtain the modal equations [20]

$$
\begin{aligned}
\frac{d \tilde{f}_{n}(t)}{d t}= & -k\left[\tilde{f}_{n}(t)-\frac{A}{2 \pi} \int_{-\pi}^{\pi} d \varphi e^{-i \varphi} \tilde{p}_{n}(\varphi, t)\right], \\
\gamma_{\perp}^{-1} \frac{\partial \tilde{p}_{n}(\varphi, t)}{\partial t}= & -\left(1-i \tilde{\alpha}_{n}\right) \tilde{p}_{n}(\varphi, t)+e^{i \alpha_{n} t} \sum_{n^{\prime}} e^{-i \alpha_{n^{\prime}} t} \tilde{f}_{n^{\prime}}(t)\left[d_{n-n^{\prime}}(\varphi, t) e^{i \varphi}+d_{n+n^{\prime}}(\varphi, t) e^{-i \varphi}\right], \\
\gamma_{\|}^{-1} \frac{\partial d_{n}(\varphi, t)}{\partial t}= & -\frac{1}{2} e^{-i \alpha_{n} t} \sum_{n^{\prime}}\left[\tilde{f}_{n^{\prime}}(t) \tilde{p}_{n^{\prime}-n}^{*}(\varphi, t) e^{i \varphi}+e^{-2 i \alpha_{n^{\prime}} t} \tilde{f}_{n^{\prime}}(t) \tilde{p}_{-n^{\prime}-n}^{*}(\varphi, t) e^{-i \varphi}+\tilde{f}_{n^{\prime}}^{*}(t) \tilde{p}_{n+n^{\prime}}(\varphi, t) e^{-i \varphi}\right. \\
& \left.+e^{2 i \alpha_{n^{\prime}} t} \tilde{f}_{n^{\prime}}^{*}(t) \tilde{p}_{n-n^{\prime}}(\varphi, t) e^{i \varphi}\right]-\left[d_{n}(\varphi, t)-\delta_{n, 0}\right] .
\end{aligned}
$$

Next, we assume conditions that allow for the adiabatic elimination of the atomic polarization (not to be confused with the rate equation approximation), i.e.,

$$
k, \gamma_{\|} \ll \gamma_{\perp}, \quad \alpha_{1} \gg \gamma_{\|} .
$$

We set the time derivative of $\tilde{p}_{n}$ equal to zero and perform a time average in Eqs. (6a)-(6c) over a time interval that is small with respect to $k^{-1}$ and $\gamma_{\|}^{-1}$ and long with respect to $\alpha_{1}^{-1}$. By substituting into Eqs. (6a) and (6c) the expression of $\tilde{p}_{n}$ found in this way, one arrives at the following set of modal equations,

$$
\begin{aligned}
\frac{d \tilde{f}_{n}(t)}{d t}= & -k\left\{\tilde{f}_{n}(t)-\frac{A}{1-i \tilde{\alpha}_{n}} \frac{1}{2 \pi} \int_{-\pi}^{\pi} d \varphi e^{-i \varphi} \tilde{f}_{n}(t)\left[d_{0}(\varphi, t) e^{i \varphi}+d_{2 n}(\varphi, t) e^{-i \varphi}\right]\right\}, \\
\gamma_{\|}^{-1} \frac{\partial d_{2 n}(\varphi, t)}{\partial t}= & -\frac{1}{2}\left\{\delta_{n, 0} \sum_{n^{\prime}} \frac{\left|\tilde{f}_{n^{\prime}}(t)\right|^{2}}{1+i \tilde{\alpha}_{n^{\prime}}}\left[d_{0}(\varphi, t)+d_{2 n^{\prime}}^{*}(\varphi, t) e^{2 i \varphi}\right]+\frac{\left|\tilde{f}_{-n}(t)\right|^{2}}{1+i \tilde{\alpha}_{-n}}\left[d_{0}(\varphi, t) e^{-2 i \varphi}+d_{-2 n}^{*}(\varphi, t)\right]\right. \\
& \left.+\delta_{n, 0} \sum_{n^{\prime}} \frac{\left|\tilde{f}_{n^{\prime}}(t)\right|^{2}}{1-i \tilde{\alpha}_{n^{\prime}}}\left[d_{0}(\varphi, t)+d_{2 n^{\prime}}(\varphi, t) e^{-2 i \varphi}\right]+\frac{\left|\tilde{f}_{n}(t)\right|^{2}}{1-i \tilde{\alpha}_{n}}\left[d_{0}(\varphi, t) e^{2 i \varphi}+d_{2 n}(\varphi, t)\right]\right\}-\left[d_{2 n}(\varphi, t)-\delta_{n, 0}\right],
\end{aligned}
$$




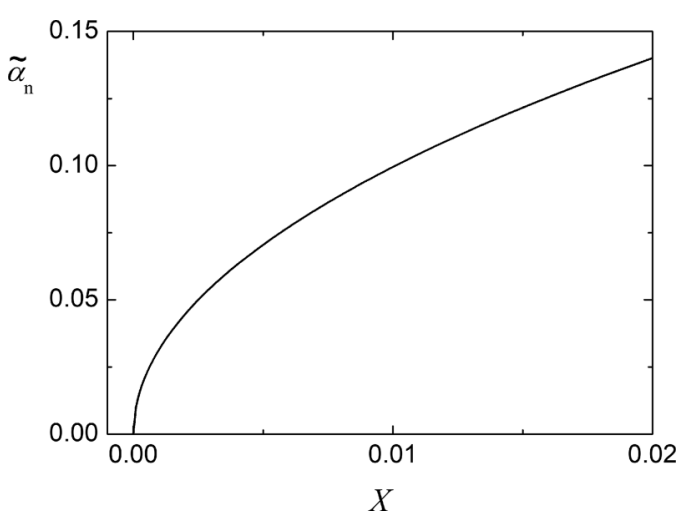

FIG. 1. Boundary of the multimode instability domain in the plane of the variables $X$ and $\tilde{\alpha}_{n}$ [see Eq. (13)]. We consider only the positive values of $\tilde{\alpha}_{n}$.

where $\tilde{\alpha}_{n}=\alpha_{n} / \gamma_{\perp}$. When $n$ is odd, $d_{n}(\varphi, t)$ obeys the trivial equation $\partial d_{n}(\varphi, t) / \partial t=-\gamma_{\|} d_{n}(\varphi, t)$ and therefore does not generate instabilities [20].

Finally, we consider a uniform (i.e., independent of $z$ ) nontrivial stationary solution of Eqs. (1a)-(1c), which corresponds to a stationary solution of Eqs. (8a) and (8b) with

$$
\tilde{f}_{n, s}=\tilde{f}_{0, s} \delta_{n, 0}, \quad d_{n, s}(\varphi)=\frac{\delta_{n, 0}}{1+4 X \cos ^{2} \varphi},
$$

and $X=\tilde{f}_{0, s}^{2}$ is obtained by inverting the relation [6]

$$
A=\frac{2 X \sqrt{1+4 X}}{\sqrt{1+4 X}-1} .
$$

If we linearize Eqs. (8a) and (8b) for $n \neq 0$ taking into account that $\tilde{f}_{n, s}$ and $d_{n, s}(\varphi)$ vanish for $n \neq 0$, we obtain the equations

$$
\frac{d \tilde{f}_{n}(t)}{d t}=-k\left[\tilde{f}_{n}(t)-\frac{A}{1-i \tilde{\alpha}_{n}} \frac{1}{2 \pi} \int_{-\pi}^{\pi} d \varphi \tilde{f}_{n}(t) d_{0, s}(\varphi)\right],
$$

$$
\frac{\partial d_{2 n}(\varphi, t)}{\partial t}=-\gamma_{\|} d_{2 n}(\varphi, t)
$$

The second equation does not lead to any instability. If we insert Eq. (9) into Eq. (11a) and integrate over $\varphi$, we get

$$
\frac{d \tilde{f}_{n}(t)}{d t}=-k\left[1-\frac{A}{1-i \tilde{\alpha}_{n}} \frac{1}{\sqrt{1+4 X}}\right] \tilde{f}_{n}(t) .
$$

If we set $\tilde{f}_{n}(t)=e^{\lambda_{n} t} \tilde{f}_{n}(0)$ and use Eq. (10), we have a multimode instability when $\operatorname{Re}\left(\lambda_{n}\right)>0$, i.e., when there is at least one pair of modes $n$ such that

$$
0 \leqslant\left|\tilde{\alpha}_{n}\right|<\sqrt{\frac{\sqrt{1+4 X}-1}{2}} .
$$

The upper boundary of the instability domain, which is shown in Fig. 1, is well approximated by $\left|\tilde{\alpha}_{n}\right|=\sqrt{X}$ for $X \ll 1$ and by $\left|\tilde{\alpha}_{n}\right|=X^{1 / 4}$ for $X \gg 1$. By taking into account that $\tilde{\alpha}_{n}=\alpha_{n} / \gamma_{\perp}$ and Eqs. (5) and (7), Eq. (13) is conveniently reformulated as

$$
\frac{\gamma_{\|} L}{\pi \tilde{c}} \ll|n|<\frac{\gamma_{\perp} L}{\pi \tilde{c}} \sqrt{\frac{\sqrt{1+4 X}-1}{2}} .
$$

It is important to note that in the case of a ring cavity the equation corresponding to Eq. (12) does not lead to any multimode instability, hence the instability defined by Eq. (13) is specific for the Fabry-Perot (FP) configuration. A remarkable feature of this instability is that it is independent of the value of the ratio $k / \gamma_{\|}$.

In order to confirm the possibility of a multimode FP instability near threshold, with the associated self-pulsing, we have solved numerically the dynamical equations (1), independent of any adiabatic elimination, for $X=0.01$ which, using Eq. (10), gives $A=1.03$, very close to the threshold $A=1$ [6]. In the numerical simulations we have considered parametric values compatible with the adiabatic elimination of the atomic polarization, i.e., with conditions (7). Furthermore, since $k / \gamma_{\perp}=T \tilde{\alpha}_{1} /(2 \pi)$ and $T$ is assumed to be much smaller than unity, we assume also that $k / \gamma_{\perp} \ll \tilde{\alpha}_{1}$.

Figure 2 gives an example of our simulations for $k / \gamma_{\perp}=$ $10^{-7 / 2}, \quad \gamma_{\|} / \gamma_{\perp}=10^{-3}$ so that $k / \gamma_{\|}=1 / \sqrt{10}, \tilde{\alpha}_{1}=0.02$. From the figure one sees the time evolution of the modal intensities, the spectrum, the self-pulsing, and the spatial profile of the total intensity at regime. We have performed similar simulations, for example, in the case $k / \gamma_{\perp}=10^{-3}$, $\gamma_{\|} / \gamma_{\perp}=10^{-7 / 2}$, which corresponds to $k / \gamma_{\|}=\sqrt{10}$, and in the case $k / \gamma_{\perp}=10^{-7 / 2}, \gamma_{\|} / \gamma_{\perp}=10^{-7 / 2}$, which corresponds to $k / \gamma_{\|}=1$. For all considered values of $k / \gamma_{\perp}, \gamma_{\|} / \gamma_{\perp}$ we have verified that there is instability for $\tilde{\alpha}_{1}=0.01,0.02, \ldots, 0.09$ and that there is no instability for $\tilde{\alpha}_{1}=0.11$, in agreement with the instability boundary shown in Fig. 1 when $X=0.01$.

Figure 2(c2) shows the frequency comb that arises in this case. Condition $\alpha_{1} \gg \gamma_{\|}$[see Eq. (7)] limits the number of lines of the comb. This condition drops in the case of adiabatic elimination of all atomic variables, that will be analyzed in Ref. [22].

As for the cavity length, if we take $n_{B}=1.5, \gamma_{\perp}>10^{12}$ (as, e.g., in solid state lasers), $L=1 \mathrm{~cm}$, we have $\tilde{\alpha}_{1} \lesssim 0.06$, hence there is instability for the value $X=0.01$ considered in our simulations. It is necessary that the population grating in the Fabry-Perot cavity is not washed out by carrier diffusion, hence edge emitting semiconductor lasers cannot be considered, and the same is true for vertical cavity surface emitting semiconductor lasers, because they are too short. An example of a device that suffers from the multimode instability described in this Rapid Communication is provided by a single section quantum dot Fabry-Perot laser (see Ref. [19], where frequency comb aspects are also discussed).

It is important to observe that the possibility of a multimode instability for a Fabry-Perot laser with values of the pump current not much higher than threshold has been demonstrated experimentally in quantum cascade lasers [25]. In this case the ratio $\gamma_{\|} / \gamma_{\perp}$ is much smaller than unity, but $\alpha_{1}$ is not much larger than $\gamma_{\|}$, so that it does not fully fit the conditions (7) considered in this Rapid Communication. This configuration will be considered in Ref. [22], also with respect to frequency combs in quantum cascade lasers [26,27].

In the case of optical bistability or a laser with an injected signal [6] there is a stationary monochromatic field injected in the cavity. Assuming that the frequency of the input field is resonant with the cavity and the atoms, these cases are described by Eqs. (1) provided that a constant term, 

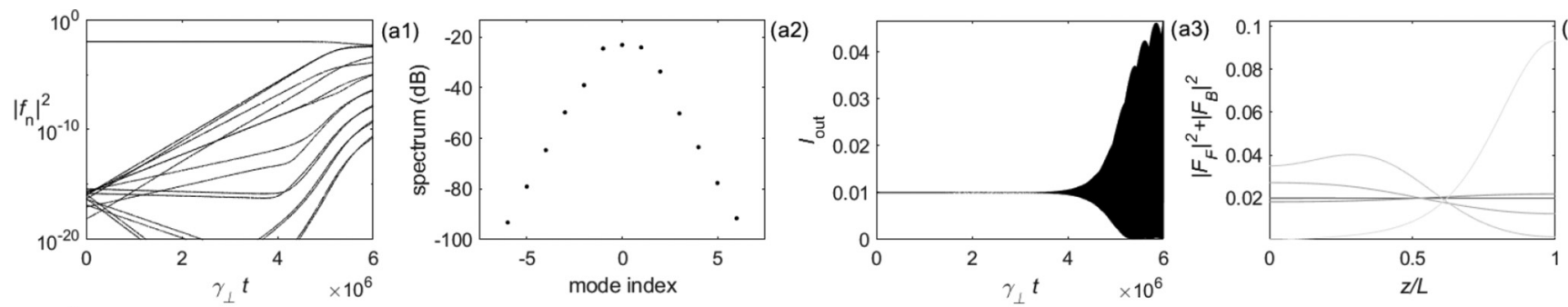

(a4)
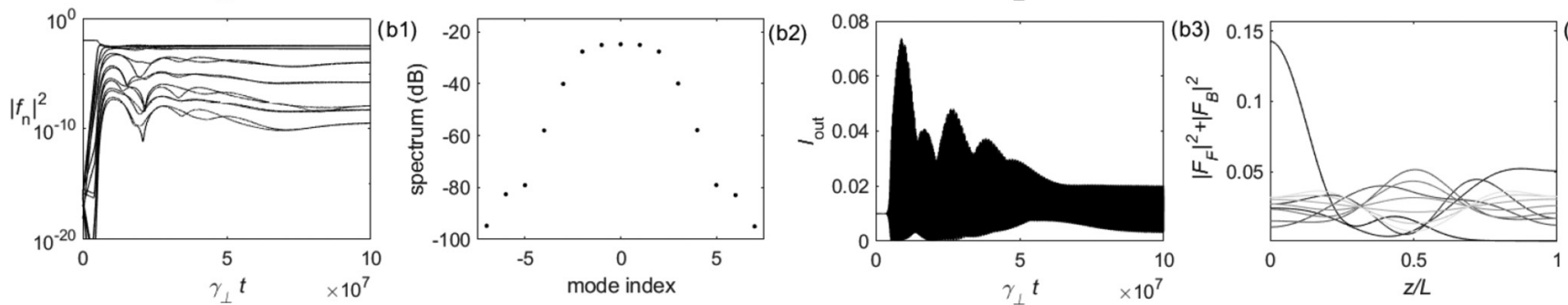

(b4)
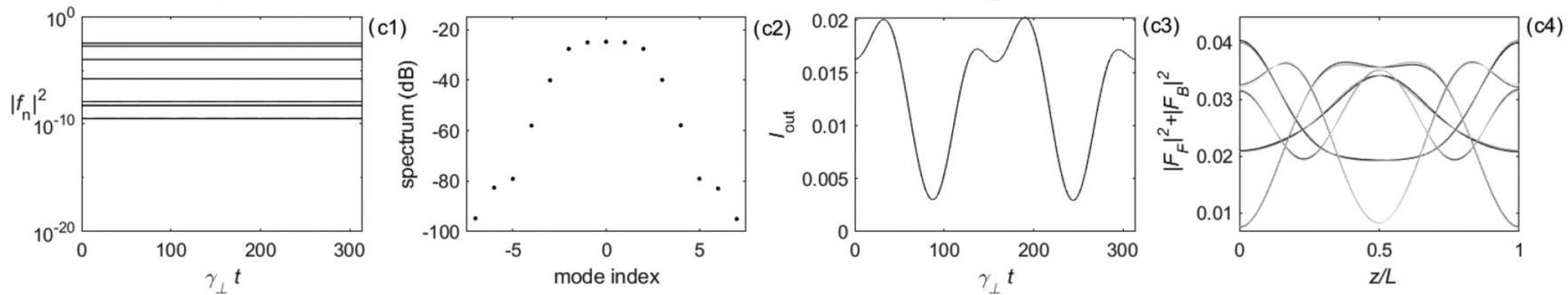

FIG. 2. The parametric values are $k / \gamma_{\perp}=10^{-7 / 2}, \gamma_{\|} / \gamma_{\perp}=10^{-3}$, so that $k / \gamma_{\|}=10^{-1 / 2}, \tilde{\alpha}_{1}=0.02$. The temporal unity is the inverse of $\gamma_{\perp}$, and the initial condition is only slightly displaced from the uniform (single-mode) stationary solution. (a1)-(a4) refer to the time evolution over the first $6 \times 10^{6}$ temporal units, during which only the intensities of the three central modes attain their stationary values. (b1)-(b4) refer to the time evolution over $10^{8}$ temporal units, during which the intensities of all other modes considered in the simulation attain their stationary values. (c1)-(c4) refer to the time evolution over the last round-trip time. (a1), (b1), and (c1) show the time evolution of the modal intensities, (a2), (b2), and (c2) show the power spectrum at the end of the simulation, (a3), (b3), and (c3) show the time evolution of the output intensity, and (a4), (b4), and (c4) show the spatial configuration of the total intensity at several instants of time, and the gray lines get lighter with time.

corresponding to the input field, is added in the square bracket in Eqs. (1a). Therefore the linearized equations (11) remain unchanged in the case of optical bistability or a laser with an injected signal, and we can ask whether the multimode instability pointed out here for a Fabry-Perot laser under conditions (7) does or does not persist in these cases.

For the laser with an injected signal Eq. (12) holds unchanged but $A$ and $X$ are independent parameters. One easily finds that there is a multimode instability for

$$
\tilde{\alpha}_{n}^{2}<\frac{A}{\sqrt{1+4 X}-1} .
$$

In the case of optical bistability the medium is an absorber instead of an amplifier, i.e., $A=-2 C$ is negative [6], with $C$ being the bistability parameter, and it is immediate to check that the instability does not persist.

Finally, it is worth noting the remarkable simplicity of formula (13) that governs the self-pulsing behavior pointed out here in the case of a free-running Fabry-Perot laser. We trust that our near-threshold instability with respect to the well-known scenario of ring laser instabilities will activate more experimental work on self-pulsing instabilities in FabryPerot lasers.
[1] P. Del'Haye, A. Schliesser, O. Arcizet, T. Wilken, R. Holzwarth, and T. J. Kippenberg, Nature (London) 450, 1214 (2007).

[2] T. Herr, V. Brasch, J. D. Jost, C. Y. Wang, N. M. Kondratiev, M. L. Gorodetsky, and T. J. Kippenberg, Nat. Photon. 8, 145 (2014).

[3] L. A. Lugiato and R. Lefever, Phys. Rev. Lett. 58, 2209 (1987).

[4] F. Castelli, M. Brambilla, A. Gatti, F. Prati. and L. A. Lugiato, Eur. Phys. J. D 71, 84 (2017).

[5] L. A. Lugiato, F. Prati, M. L. Gorodetsky, and T. J. Kippenberg, Philos. Trans. R. Soc. A 376, 20180113 (2018).
[6] L. A. Lugiato, F. Prati, and M. Brambilla, Nonlinear Optical Systems (Cambridge University Press, Cambridge, UK, 2015).

[7] Y. K. Chembo, Nanophotonics 5, 214 (2016).

[8] H. Risken and R. Nummedal, J. Appl. Phys. 39, 4662 (1968).

[9] R. Graham and H. Haken, Z. Phys. 213, 420 (1968).

[10] E. Roldán, G. J. de Valcárcel, F. Prati, F. Mitschke, and T. Voigt, in Trends in Spatiotemporal Dynamics in Lasers. Instabilities, Polarization Dynamics, and Spatial Structures, edited by O. Gomez-Calderón and J. M. Guerra (Research Signpost, Trivandrum, India, 2005), pp. 1-80. 
[11] N. B. Abraham, P. Mandel, and L. M. Narducci, in Progress in Optics, edited by E. Wolf (North-Holland, Amsterdam, 1988), Vol. 25, p. 1.

[12] P. Mandel, J. Opt. Soc. Am. B 2, 112 (1985).

[13] C. L. Tang, H. Statz, and G. deMars, J. Appl. Phys. 34, 2289 (1963).

[14] P. Mandel, Theoretical Problems in Cavity Nonlinear Optics (Cambridge University Press, Cambridge, UK, 1997).

[15] K. Otsuka, Prog. Quantum Electron. 23, 97 (1999).

[16] P. Mandel, Eur. Phys. J. D 8, 431 (2000).

[17] T. Hill, M. W. Hamilton, D. Pieroux, and P. Mandel, Phys. Rev. A 66, 063803 (2002).

[18] M. Homar, J. V. Moloney, and M. San Miguel, IEEE J. Quantum Electron. 32, 553 (1996).

[19] P. Bardella, L. L. Columbo, and M. Gioannini, Opt. Express 25, 26234 (2017).

[20] L. A. Lugiato and F. Prati, Phys. Scr. 93, 124001 (2018).
[21] The model provided by Eqs. (1) is substantially simpler than the traveling wave models for multimode Fabry-Perot lasers considered in Refs. [18,19], which involve also the backward propagating envelope.

[22] L. A. Lugiato and F. Prati (unpublished).

[23] H. J. Carmichael, Opt. Commun. 53, 122 (1985).

[24] L. A. Lugiato and L. M. Narducci, Z. Phys. 71, 129 (1988).

[25] T. S. Mansuripur, C. Vernet, P. Chevalier, G. Aoust, B. Schwarz, Feng Xie, C. Caneau, K. Lascola, C.-E. Zah, D. P. Caffey, T. Day, L. J. Missaggia, M. K. Connors, C. A. Wang, A. Belyanin, and F. Capasso, Phys. Rev. A 94, 063807 (2016).

[26] A. Hugi, G. Villares, S. Blaser, H. C. Liu, and J. Faist, Nature (London) 492, 229 (2012).

[27] Q. Y. Lu, M. Razeghi, S. Slivken, N. Bandyopadhyay, Y. Bai, W. J. Zhou, M. Chen, D. Heydari, A. Haddadi, R. McClintock, M. Amanti, and C. Sirtori, Appl. Phys. Lett. 106, 051105 (2015). 